\title{
Pratiques
}

Linguistique, littérature, didactique

\section{Linguistique populaire, language awareness, linguistique appliquée : interrelations et transitions}

\section{Martin Stegu}

\section{(2) OpenEdition}

\section{Journals}

Édition électronique

URL : http://journals.openedition.org/pratiques/1193

DOI : 10.4000/pratiques. 1193

ISSN : 2425-2042

Éditeur

Centre de recherche sur les médiations (CREM)

\section{Édition imprimée}

Date de publication : 15 décembre 2008

Pagination : 81-92

Référence électronique

Martin Stegu, «Linguistique populaire, language awareness, linguistique appliquée : interrelations et transitions », Pratiques [En ligne], 139-140 | 2008, mis en ligne le 15 décembre 2008, consulté le 08 mars 2021. URL : http://journals.openedition.org/pratiques/1193 ; DOI : https://doi.org/10.4000/ pratiques. 1193 


\section{Linguistique populaire, language awareness, linguistique appliquée : interrelations et transitions}

\section{Martin Stegu}

Wirtschaftsuniversität Wien

\section{Introduction}

Dans le cadre de ce recueil consacré à divers aspects de la « linguistique populaire », la contribution présente poursuit les objectifs suivants :

a. Analyser et discuter les rapports qui existent entre la « linguistique populaire » et d'autres approches potentiellement affines, dont, plus particulièrement, la conscience linguistique (language awareness) ;

b. Adopter une perspective contrastive et intégrer, d'un point de vue onomasiologique et sémasiologique, les recherches provenant des pays germanophones (en discutant également les termes «Volkslinguistik» et « Laienlinguistik »);

c. Étudier les rapports - réels et potentiels - entre la linguistique populaire et la linguistique appliquée (en tant que métadiscipline, mais aussi ses différentes sous-disciplines).

Dans cette contribution, il ne s'agit pas seulement d'un effort de synthèse consistant à comparer des notions bien définies et préexistantes : de la même façon que «la » linguistique populaire n'existe pas comme concept homogène et établi, il n'y a pas non plus un concept unique de «language awareness » accepté par la totalité de la communauté des linguistes. Nous devrons donc aussi analyser et discuter chacun des termes traités individuellement, en essayant d'emprunter un chemin non-dogmatique et pluraliste.

Dans une première partie (1.), nous allons donc discuter les rapports qui existent entre la linguistique populaire et la linguistique officielle. Puis (2.), nous allons comparer l' expression « linguistique populaire» avec les variantes allemandes «Laienlinguistik» et «Volkslinguistik» (en opposant cette dernière expression à son pseudoéquivalent anglais " folk linguistics »); ensuite, dans une troisième partie (3.), nous allons présenter le concept - ou plutôt les concepts - qui se cache(nt) derrière l'étiquette « language awareness » et ses synonymes et équivalents potentiels en anglais, en français et en allemand. Nous mentionnerons également des « consciences partielles » et des « consciences mixtes», comme «pho- 
nological awareness », « semantic awareness », « language learning awareness », «plurilingual awareness » et des concepts apparentés, comme « intercultural awareness », " communication awareness » etc. La fin de cette troisième partie sera consacrée aux relations réciproques entre la conscience linguistique (à savoir, en première ligne, la conscience linguistique explicite) et la linguistique populaire. Notre quatrième partie (4.) traitera enfin des rapports existant entre la linguistique populaire et la linguistique appliquée (ainsi que ses différentes sous-disciplines).

\section{Linguistique officielle $v$ s linguistique populaire}

Dans le contexte francophone, c'est avant tout Marie-Anne Paveau qui plaide pour une revalorisation du rôle de la linguistique populaire dans la discussion linguistique « officielle» (voir Paveau 2006, 2007); elle se prononce également en faveur du terme même de « linguistique populaire », qu'elle préfère à ceux de « linguistique spontanée » ou « naïve » ou « de sens commun» (voir ici même), malgré la polysémie du mot «populaire » et ses connotations potentiellement péjoratives.

On pourrait se demander d'ailleurs si la plupart des linguistes ont vraiment l'intention d'exclure toute connotation péjorative de cette expression; même pour les quelques linguistes qui prennent au sérieux la linguistique populaire, celle-ci n'aura jamais le même statut épistémologique ou « scientifique » que la linguistique officielle.

Même si on ne veut pas adopter une attitude postmoderne radicale ( $c f$. Stegu 1998), qui, avec son pluralisme poussé à l'extrême, attribuerait la même valeur et le même statut à toute théorie ou à toute conviction etc., qu'elle soit scientifique ou non-scientifique, la situation me paraît pourtant assez complexe. D'abord, il ne faut pas oublier qu'il n'existe ni une version unique de la linguistique officielle ni une seule variante de la linguistique populaire. Il y a un nombre potentiellement illimité de théories linguistiques, scientifiques ainsi que populaires - indépendamment de la ligne de démarcation supposée entre ces deux domaines. Et des deux côtés, il y a de grandes différences de qualité, et on ne peut pas généraliser et qualifier toutes les théories officielles de «bonnes » et toutes les théories populaires de «mauvaises ».

Quels sont d'ailleurs les critères qui distinguent une théorie populaire d'une théorie non populaire ? J'utilise ici « non populaire », parce que la dénomination de cet antonyme n'est pas non plus évidente - mon propre choix ( « scientifique») est seulement provisoire, et il faudra procéder à une recherche basée sur corpus pour voir quels sont les termes les plus utilisés (et quels seraient les plus adéquats). Pour beaucoup de linguistes, le contraire de «linguistique populaire » sera la « linguistique » tout court; mais dès lors qu' on attribue une certaine importance aussi à des théories « populaires », il faudra trouver une terminologie pour désigner les théories «non populaires».

Ces réflexions ne s'appliquent pas seulement à la linguistique, mais à toutes les autres disciplines où il y a un parallélisme de recherches scientifiques et de convictions populaires. Le grand défi pour la linguistique consiste, cependant, à pouvoir servir également de métadiscipline, analysant et décrivant les pratiques discursives qui distinguent la recherche « officielle » d'approches populaires, à un niveau très général, mais aussi et surtout quant à son propre discours disciplinaire autoréférentiel - c'est-à-dire, par quels moyens discursifs et argumentatifs la linguistique officielle se démarque de la linguistique populaire. 
Pour revenir sur les critères de différenciation entre « caractère scientifique » et « caractère populaire», nous pourrions distinguer des critères externes et internes. Parmi les critères internes on mentionnera la cohérence logique de l'argumentation, l'absence de contradictions, l'observation de certaines règles formelles de citation etc. Il s'agit là avant tout de critères normatifs, lesquels sont partiellement problématiques (puisque par exemple pour les sciences humaines et pour les sciences naturelles, les règles discursives ne sont pas identiques, et différentes écoles sont aussi souvent caractérisées par leurs langages différents).

Quant aux critères externes (ou sociaux), il faut surtout mentionner l'habilitation des personnes qui publient des textes « scientifiques » (ou qui en font des présentations orales) : on s'attend à ce qu'une personne disposant d'une certaine formation (normalement universitaire) et, en plus, d'une position dans une institution de recherche, garantisse ex officio la production de textes de caractère «scientifique » et non pas «populaire ». Dans une situation idéale (ou idéalisée) il y a complémentarité totale entre critères externes et internes, lorsque, par exemple, un professeur des universités publie un article selon les règles textuelles et discursives de sa communauté scientifique, ou, dans le cas opposé, un non-linguiste écrit une lettre à un journal où il se prononce, plein d'émotions, contre l'influence croissante des anglicismes.

Mais il ne faut pas perdre de vue que les critères externes et internes sont de nature différente et qu'ils ne sont pas automatiquement et à 100\% reliés entre eux.

L'idée d'une ligne de démarcation nette séparant le « scientifique » du «populaire » me semble être également une construction - ou illusion - normative, soit dans le domaine des critères internes (pensons à des œuvres didactiques et «popularisantes »), soit quant aux critères externes : la différence entre spécialistes et non-spécialistes est graduelle ( $c f$. Stegu 2007). Un professeur de linguistique - et supposons de plus, un syntacticien - prenant position, dans une discussion à la télévision, pour ou contre les anglicismes - représente-t-il dans ce cas la linguistique officielle ou populaire ? Un enseignant de langue(s) dans un lycée est-il un spécialiste en linguistique, un non-spécialiste ou bien se trouve-t-il entre les deux?

Une prise en compte de la linguistique dite populaire dans le discours de la linguistique officielle ne signifie donc pas encore une revalorisation de celle-là ; au contraire, parfois cette prise en compte peut servir à faire ressortir encore mieux les (prétendues ?) grandes différences entre les deux approches. Selon moi, il est certain que la plupart des théories populaires n'ont pas le même standard ou la même qualité que les théories officielles ; mais cela ne nous permet pas d'identifier « populaire » a priori avec « incorrect» ou « mauvais» ni à considérer la frontière entre le «scientifique » et le «populaire » comme un phénomène prédonné, stable et hors toute problématisation. N'oublions pas que beaucoup d'éléments des savoirs que nous qualifions aujourd'hui de "populaires » faisaient partie de la doxa officielle il y a encore quelque temps.

Comme il s'agit ici plutôt d'une présentation générale et d'une vue d'ensemble, également sur le plan terminologique, je n'ai pas l'intention de proposer moi-même des définitions nouvelles, elles-mêmes forcément normatives. Mais j'ai constaté que, au moins, dans les domaines de la linguistique populaire et de la conscience linguistique, beaucoup d'auteurs choisissent dès le début des définitions restrictives qui excluent des aspects qui seraient également très importants. Achard-Bayle et Paveau (ici même, p. 5) définissent " populaire » comme « le savoir spontané des acteurs sur le monde (déposé dans les proverbes ou dictons par 
exemple), qui se distingue du savoir savant ou scientifique comme le savoir-faire se distingue du "savoir que" et le sens commun du savoir scientifique ». Mis à part le fait que le « savoir populaire ", à mon sens, est pour une grande partie aussi un «savoir que», je m' interroge sur les raisons de limiter ce savoir (et donc la linguistique) populaire à un savoir spontané. Et même si les proverbes, les dictons etc. sont sans aucun doute très importants pour comprendre certains aspects du savoir populaire, je ne m'y arrêterai pas non plus.

À mon avis, le savoir populaire comprend des domaines spontanés et non spontanés, et je proposerais de partir d'une définition de base qui n'exclue pas de prime abord une grande partie de phénomènes très apparentés pour lesquels il faudra trouver par la suite également un terme adéquat (plus un hyperonyme général qui couvre le champ sémantique dans sa totalité). Le savoir populaire serait donc tout le savoir implicite et explicite, spontané ou « élicité » à repérer chez les « nonscientifiques » et les « non-spécialistes » (ou même chez les « scientifiques » etc., quand il s'agit d'un domaine qui ne fait pas immédiatement partie de leur spécialisation).

On pourrait se demander s'il faut tenir compte du savoir implicite et du savoir explicite dans la même mesure. D'un point de vue "germanophone », on aurait plutôt tendance à exclure l'aspect implicite, parce que la sémantique de « wissen » se réfère beaucoup plus à l' explicite que les « équivalents » français et anglais «savoir » et « to know ». Mais comme on s'est prononcé pour des définitions (et des approches) larges, on ne va donc pas exclure le savoir implicite, d'autant plus que les frontières entre l'implicite et l'explicite sont, elles aussi, assez floues.

Quant au «savoir linguistique », qui nous intéresse tout particulièrement, je vois une solution terminologique en intégrant la notion de «language awareness». La conscience linguistique - qui sera traitée de manière plus détaillée dans la partie 3. - désignerait tout phénomène cognitif implicite ou explicite, ayant trait au langage (à la langue, aux langues etc.), tandis que le terme « linguistique populaire » devrait se référer seulement à des réflexions explicites et (quasi- ?) théoriques, en général, de non-linguistes. La « linguistique officielle», d'ailleurs, est à mon sens également une sous-catégorie de la «language awareness », de sa partie explicite, bien sûr, et se situe juste à côté de la linguistique populaire, avec des frontières potentiellement « déplaçables ».

Linguistique populaire et linguistique scientifique sont des aires très imbriquées entre elles, comme c'est le cas aussi dans d'autres domaines du savoir. Quand par exemple quelqu'un est toujours persuadé que c'est le soleil qui tourne autour de la terre, et non pas vice versa, il faudrait catégoriser cette conviction comme «populaire »; mais le contraire de cette conviction n'est pas (ou n'est plus) exclusivement « scientifique », elle est, de nos jours, parallèlement et scientifique et populaire.

Je voudrais insister encore une fois sur le fait que la classification comme « scientifique» $v s$ «non scientifique», «non populaire» $v s$ «populaire» n'est pas totalement objective ou inhérente à la discipline concernée. Beaucoup de linguistes se disputeront par exemple sur la question de savoir si l'assertion « Dans la langue X il y a trop d'anglicismes. » est plutôt populaire ou scientifique. Ou pour un certain groupe de linguistes, la pragmatique et, encore plus, la stylistique et (la plupart des sous-disciplines de) la linguistique appliquée se trouvent au-delà ou plutôt en deçà du "scientifique ", et donc très proches d'un type de savoir populaire. Cependant, le caractère problématique de leur délimitation réciproque ne devrait pas mettre en cause le principe même d'opérer une distinction entre ces deux catégories. 
Parmi les expressions françaises déjà utilisées ou potentiellement disponibles pour la « linguistique de non-spécialistes » le terme de «linguistique populaire» me paraît en effet être le plus adéquat; de toute façon, il est beaucoup plus général que " linguistique spontanée » et beaucoup moins péjoratif que « linguistique naïve », pour ne reprendre que deux des termes mentionnées par Marie-Anne Paveau (ici même). Pour certains locuteurs d'autres langues que le français, il est vrai, la connotation «(anglais :) popular / (allemand :) populär = très apprécié par un large public » risque d'interférer et d'irriter un peu, d'autant plus que la linguistique (scientifique) compte malheureusement toujours parmi les disciplines les plus inconnues en dehors de la communauté même des linguistes ...

\section{Laienlinguistik, Volkslinguistik, folk linguistics}

Dans cette partie nous allons discuter les termes allemands « Laienlinguistik» et «Volkslinguistik»; en outre, nous allons comparer les aspects sémantiques dénotatifs et connotatifs de ce dernier terme à ceux de son équivalent étymologique anglais « folk linguistics».

C'est en 1996 que le germaniste allemand Gerd Antos a publié sa « thèse d'habilitation » intitulée « Laien-Linguistik» (Antos 1996). Ce livre et surtout son titre sont devenus très vite connus dans les pays germanophones, ce qui est entre autres dû à la notoriété d'Antos, qui a été pendant très longtemps membre du directoire et même président de la «GAL » (= «Gesellschaft für Angewandte Linguistik », la société allemande de linguistique appliquée).

«Laie» en allemand signifie d'abord « lai », «profane » au sens religieux, mais surtout «non-spécialiste», et dans un mot composé comme celui-ci, aucun germanophone n'aurait d'associations religieuses. Pour l'allemand, ce terme me paraît être le plus neutre parmi les expressions potentiellement concurrentielles (surtout par rapport à « Volkslinguistik », voir plus bas) et il se prête aussi à désigner un « concept-toit» qui contiendrait plusieurs sous-catégories.

Le sous-titre du livre d'Antos s'intitule «Studien zu Sprach- und Kommunikationsproblemen im Alltag. Am Beispiel von Sprachratgebern und Kommunikationstrainings " ("Études sur les problèmes de langue et de communication au quotidien. Exemples pris dans les guides de rédaction et dans les formations à la communication ») et il nous présente son objet d'études central : les manuels et les formations qui promettent - surtout au locuteur natif une amélioration de sa compétence langagière et communicative. Pour Antos, la Laien-Linguistik est une linguistique destinée aux non-linguistes, mais aussi très souvent pratiquée par des non-linguistes (Antos 1996, p. 3), puisque beaucoup de formateurs sont des psychologues, des spécialistes de management ou simplement des autodidactes.

On pourrait reprocher à Antos de prévoir un titre tellement général et de se contenter en fin de compte d'un phénomène, il est vrai, très intéressant et pertinent, mais cependant assez limité. Une telle critique ne serait que partiellement justifiée. Comme il s'agit d'une thèse d'habilitation, le livre contient une partie théorique très solide discutant également les rapports avec la «Volkslinguistik » ainsi qu'avec les « lay theories » de Furnham (1988), qui sont à la base de la création du terme « Laien-Linguistik».

Furnham établit toute une liste de différences entre les «lay theories » et les théories scientifiques, bien qu'il se prononce aussi pour une interprétation graduelle de ces différences (Furnham 1988, p. 8, voir aussi Antos 1996, p. 34). Pour 
Antos la différence principale consiste dans la systématicité de l'approche scientifique (voir Antos 1996, p. 34). C'est certainement vrai, mais le problème persiste pour l'analyse de théories partielles ou de fragments de théories (par exemple d'hypothèses), de savoir si on réussit à reconnaître immédiatement d'après leur forme ou qualité leur origine « de non-spécialiste».

Même si on est d' accord avec le principe de la transition graduelle entre le scientifique et le non-scientifique et avec l'impossibilité de classifier une théorie populaire comme « a priori fausse », on est toujours confronté à la question centrale de savoir quels sont les rapports existant entre «le degré de scientificité » et «le degré de "popularité" / "layness" " d'un côté et la qualité d'une théorie de l'autre. Pendant toute la lecture du livre d'Antos on n'est pourtant jamais sûr jusqu'à quel point et sous quelles conditions l'auteur serait prêt à accepter les théories populaires qu'il présente et quand par contre il préférerait décidément des théories linguistiques scientifiques.

Antos est très conscient du problème des prescriptions et de la normativité dans le cadre de la linguistique appliquée (voir Antos 1996, pp. 19 sq.), et il n'a donc aucunement l'intention d'écrire lui-même un livre prescriptif. Mais quand un livre «scientifique » décrit des phénomènes non ou, à la rigueur, parascientifiques, l'aspect de la norme ou des normes dans les sciences devrait être intégré de façon plus explicite - non pas en postulant des normes définitives, certes, mais en les discutant et en les problématisant ouvertement.

Avant de passer à la « Volkslinguistik », je voudrais encore mentionner un autre domaine de la linguistique appliquée, dans lequel on a analysé des opinions «populaires » et qu'on associe dans les pays germanophones à un livre qui a paru la même année que celui d'Antos - il s'agit de la thèse de Christiane Kallenbach (1996) : «Subjektive Theorien : Was Schüler und Schülerinnen über Fremdsprachenlernen denken », où l'auteure étudie les opinions «populaires »d'élèves de lycée à propos de l'apprentissage de langues étrangères et surtout à propos de leurs propres expériences dans ce contexte.

Cette approche se base également sur Furnham, sur des travaux qui étudient le « sens commun » et la réalité de 1' «Alltag» (= « la réalité de tous les jours »), par exemple dans la tradition de Schütz 1932, mais aussi et surtout sur les projets menés par le psychologue Groeben (par exemple Groeben et al. 1988), qui a également créé la paire terminologique de théories objectives (= scientifiques) et subjectives. Kallenbach voit d'ailleurs une ligne de démarcation assez nette entre ces deux types de théories.

L'expression «théories subjectives » paraît jusqu'à un certain degré synonyme de celui de «théories populaires », mais met un accent plus fort sur des expériences personnelles.

Malgré le grand profit qu'on peut tirer de la lecture de ce livre, on ne comprend pas très bien quelle a été la motivation pour analyser les théories subjectives de ces élèves (sur la base d'interviews de 17 personnes) : s'agit-il d'un intérêt primordialement intellectuel ou s'attend-on à une amélioration des processus d'apprentissage ? Et quel est le rapport entre les différentes formes de théories subjectives et les compétences langagières des élèves interviewés?

Le même type de questions se pose sous une forme ou sous une autre pour toute la linguistique appliquée : quand faudrait-il se contenter d'une pure analyse descriptive, et quand faudrait-il intervenir pour changer et améliorer les convictions ainsi que les pratiques communicatives qui en résultent?

Quant au terme «Volkslinguistik», on le doit surtout à deux articles de Herbert 
Brekle (1985, 1986 ; il existe d'ailleurs aussi une version française 1989). Depuis cette époque, cet auteur n'a plus travaillé sur ce sujet. Chez Brekle on peut constater le même phénomène qu'on vient de trouver chez d'autres auteurs : Il ne prévoit pas le terme "Volkslinguistik » dans un sens large, mais seulement pour désigner des expressions et des énoncés qui provoquent chez les partenaires de la communication «des effets d'une pertinence sociale et pratique» (« um damit bei Kommunikationsteilnehmern bestimmte gesellschaftlich-praktisch relevante Wirkungen hervorzubringen », Brekle 1985, 145). Ainsi, il exclut des énoncés qui sont produits juste pour contribuer à un «gain de connaissances », puisque, dans ce cas, il s'agirait déjà d' "énoncés linguistiques » ("sprachwissenschaftliche Aussagen », Brekle 1985, 145), se basant au moins sur des « Minimalkriterien » scientifiques. Cela veut dire que Brekle procède à une catégorisation un peu idiosyncratique, puisque les « lay theories », qui nous intéressent tout particulièrement, sont, en effet, exclues de sa «Volkslinguistik ». La «Volkslinguistik» de Brekle est donc plus proche de la conception d'un « métalangage» non théorique, ce qui nous rappelle d'ailleurs la nécessité d'intégrer aussi le terme «métalangage » dans nos réflexions terminologiques générales.

Bien sûr, nous ne sommes pas obligés de respecter les contraintes de Brekle, nous pourrions aussi proposer le terme «Volkslinguistik» soit exclusivement pour les théories (ou fragments de théories) linguistiques de non-spécialistes soit - comme hyperonyme - pour les énoncés métalinguistiques non / a-théoriques et (pré-) théoriques. Les lecteurs devineront que nous plaiderions plutôt pour une sémantique plus large qui pourrait intégrer tous les phénomènes apparentés pertinents, sans pourtant exclure une subcatégorisation plus fine par la suite.

Si nous libérons la «Laienlinguistik» et la «Volkslinguistik» des contraintes sémantiques prévues implicitement ou explicitement par des auteurs comme Antos et Brekle, nous obtenons deux termes potentiellement synonymes - le premier nous fait penser aux « lay theories », le second évoque le terme anglais de " folk linguistics ». Comme le mot «Volk» en allemand n'est toujours pas libre de connotations problématiques dues aux moments les plus sombres de l'histoire des pays germanophones, il me paraît plus raisonnable de se servir de l'expression « Laienlinguistik » comme équivalent de «folk linguistics » et de «linguistique populaire».

\section{Language awareness}

Le « language awareness movement» est né dans les années 80 en Grande Bretagne quand les autorités britanniques ont constaté un manque de compétence langagière chez une grande partie des élèves de l'école primaire (voir Hawkins 1987). Une certaine sensibilisation linguistique - pour des aspects structurels, mais aussi et surtout pour des aspects pragmatiques - devait contribuer à améliorer les compétences langagières et communicatives principalement en anglais comme « L1» (=traditionnellement appelée «langue maternelle»). Bientôt le concept a été aussi reçu et répandu par la didactique des langues étrangères (voir James / Garrett 1992), dans le contexte d'un tournant cognitif (et aussi réflexif), comme réponse à une orientation purement behavioriste (et aussi exclusivement « communicativiste »). En dehors du domaine didactique, le terme joue aussi un rôle dans la sociolinguistique, surtout dans des constellations multilingues : dans quelle mesure les locuteurs sont-ils conscients de leur propre L1 (souvent une langue minoritaire) et des autres langues parlées dans leur région ou dans leur pays (voir Cichon 2005) ? Ces deux types d'intérêt se sont potentiellement rapprochés ces derniers 
temps, en conséquence de l'actualité grandissante du plurilinguisme européen et du développement d'une didactique de plus en plus plurilingue (Meißner 2003, Meißner, Reinfried 1998).

Selon la définition de Donmall (1985 : 7), language awareness est « a person's sensitivity to and conscious perception of the nature of language and its role in human life ». Sur une version antérieure de sa page web - mais toujours consultable l' "Association for Language Awareness » donne la définition suivante : «Language Awareness can be defined as explicit knowledge about language, and conscious perception and sensitivity in language learning, language teaching and language use. » (en ligne sur http ://www.lexically.net/ala/ la_defined.htm).

Comme il s'agit d'une notion assez récente, on peut s'attendre moins encore à des définitions précises et univoques de «language awareness » que dans d'autres cas de la terminologie linguistique. Pour gagner une image aussi complète que possible du champ sémantique en question, il faudrait procéder à une analyse sémasiologique (à partir d'expressions comme « language awareness », « linguistic awareness », " metalinguistic awareness » repérer les concepts-clés se trouvant derrière), onomasiologique (à partir des concepts identifiés rechercher encore d'autres signifiants correspondants potentiels) et contrastive (intégrant des termes apparentés comme «noticing» [voir Schmitt 1995, Robinson 1995], «éveil aux langues » [voir Candelier 2003], "Sprachbewusstheit », "Sprachbewusstsein », «Sprachreflexion» [voir Gnutzmann 2003], etc.).

Dans le composé « language awareness », les deux éléments peuvent être interprétés de plusieurs façons, ce qui peut entraîner plusieurs (sous-) types de conscience linguistique qui seraient tous justifiables. "Language » peut être vu comme " langage », comme notre instrument de communication le plus puissant (malgré certaines limites), mais aussi comme « langue (concrète)», comme structure ou système ou bien dans son usage; le terme peut se référer également à plusieurs langues et se transformer en une «multilingual awareness ». On peut également présumer des consciences partielles, comme «phonological awareness », « semantic awareness », « style awareness », « norm awareness » (Stegu 2008a), etc., mais aussi des formes mixtes ou complexes comme «language learning awareness » (voir Edmondson 1997, Knapp-Potthoff 1997, Chryshochoos 1992), «multi- / plurilingual awareness » (voir Morkötter 2005, Jessner 2006), « code switching awareness » (Stegu 2008b), etc. On pourrait ouvrir le terme « language » vers des consciences apparentées telles que « communication awareness », " semiotic awareness », « cultural / intercultural awareness » (Tomalin, Stemplewski 1993), etc.

Quant à l'élément « awareness » (partiellement en concurrence avec «consciousness »), on a à voir à deux différentes vues principales : ou bien on oppose «awareness » aux théories explicites, comme pure sensibilité vis-à-vis de certains phénomènes, ou bien on prévoit deux pôles d'une conception très large de « awareness » qui mène de l'implicite à l'explicite (voir aussi les deux définitions présentées plus haut). Dans cette dernière conception, les théories populaires ainsi que scientifiques feraient toutes les deux partie (ou seraient un reflet) d'une certaine conscience linguistique. En général, par contre, le noyau prototypique de « language awareness » sera la conscience linguistique populaire, en l'occurrence celle des non-linguistes.

Selon moi - défendant une fois de plus les définitions primaires larges - on ne peut pas totalement séparer 1' " awareness » même dans son sens de « sensibilisation » des théories populaires qui sont souvent à leur base et qui peuvent les influen- 
cer considérablement. Ainsi, quand on est par exemple « interculturellement » sensible, cette sensibilité est presque toujours combinée avec certaines idées théoriques à propos de «culture(s) » et « interculturalité ». Il paraît impensable par ailleurs d'exprimer certaines attitudes (voir Garrett, Coupland 2003) - terme à rapprocher de notre conception très large de l'« awareness » - face à des performances «non correctes», sans posséder une théorie au moins fragmentaire et sousjacente relative au rôle d'une norme linguistique.

Chez les différents auteurs on trouve des définitions très diverses de «language awareness », et il y a aussi beaucoup de concepts apparentés avec des désignations très variées. Pour le contexte français, il faudra par exemple encore mentionner le mouvement de l' " éveil aux langues ", grâce auquel on veut sensibiliser surtout de jeunes enfants à la réalité plurilingue de notre monde (voir Candelier 2003).

Selon moi, « language awareness » est - aussi sur le plan international-1e terme le plus répandu et se prête donc aussi à un rôle de nom générique pour un ensemble de phénomènes similaires, probablement tous reliés entre eux. Dans ce cas, la linguistique populaire ferait partie de ce concept très large, comme reflet ou soustype de ce phénomène et marqué par son caractère explicite et verbalisé, mais sans reconnaissance officielle et / ou scientifique. Même si on n'accepte pas une conception aussi large de «language awareness », il y a de fortes affinités entre celleci et la linguistique populaire puisque les théories populaires ont des impacts sur la sensibilité linguistique et vice versa.

\section{La linguistique populaire et la linguistique appliquée}

La linguistique appliquée n'est pas une discipline homogène, mais un ensemble de sous-disciplines, voire de "sous-inter-disciplines » qui ont pour objectif de contribuer à résoudre des problèmes langagiers et communicatifs réels. Ainsi, Brumfit (1997 : 93), dans sa définition célèbre, voit la linguistique appliquée comme " theoretical and empirical investigation of real-world problems in which language is a central issue ». La définition présentée par l'AILA (= Association Internationale de Linguistique Appliquée) décrit de façon plus détaillée les différents sous-domaines dans une liste potentiellement ouverte : "The problems Applied Linguistics deals with range from aspects of the linguistic and communicative competence of the individual such as first or second language acquisition, literacy, language disorders, etc. to language and communication related problems in and between societies such as e.g. language variation and linguistic discrimination, multilingualism, language conflict, language policy and language planning. » (En ligne sur http ://www.aila2008.org/applied-linguistics.html)

Pour caractériser la linguistique appliquée contemporaine, il faut souligner qu'il ne s'agit pas seulement de quelques aspects appliqués de la linguistique théorique, mais d'approches indépendantes, qui, bien que se basant souvent sur des éléments de théories linguistiques, développent aussi des théories autonomes, très souvent de caractère interdisciplinaire. Comme au début la linguistique appliquée s'intéressait surtout à l'apprentissage de langues secondes et étrangères, elle s'est ouverte de plus en plus vers toutes sortes de problèmes de communication que nous pouvons rencontrer dans nos sociétés actuelles.

La linguistique populaire ne peut certainement pas être considérée comme sous-discipline de la linguistique appliquée - mais il y a plusieurs zones où les deux domaines se rapprochent beaucoup. Même si la linguistique théorique peut, 
elle aussi, s'intéresser à la linguistique populaire (pour étudier les différences synchroniques et diachroniques existant entre les théories officielles et populaires), la linguistique appliquée montre a priori plus d'affinités avec la linguistique populaire. La linguistique appliquée est une linguistique conçue et développée prioritairement pour les non-linguistes, et c'est ainsi que cette linguistique doit tenir compte des connaissances et des croyances ("populaires») de cette cible. Et si on peut et veut influencer ces convictions on passera justement par la linguistique appliquée. Et s'il y a des frontières ouvertes, on en trouvera aussi plutôt entre la linguistique appliquée et la linguistique populaire qu'entre la linguistique théorique (actuelle) et cette dernière. Rappelons à cet égard les manuels de « LaienLinguistik » analysés par Antos qui se trouvent dans une zone floue entre linguistique appliquée « officielle » et populaire.

Pour améliorer les effets potentiels d'une linguistique appliquée qui vise à influencer les pratiques communicatives réelles, il faudra encore prendre plus au sérieux les croyances populaires pour mieux y réagir.

On pourrait d'ailleurs se demander si les « convictions » transportées par la linguistique appliquée réussissent à remplacer les convictions populaires des nonlinguistes. Et pour adapter une question posée au début : dans ce cas, ne s'agirait-il alors plus de théories populaires ou simplement de théories populaires améliorées ? De toute façon, on pourra supposer que les nouvelles variantes de ces théories populaires représenteront une synthèse des théories officielles et des théories populaires originales. Dans la plupart des cas, les théories linguistiques populaires ne seront jamais purement populaires - il s'agira presque toujours de conglomérats d'éléments scientifiques, absorbés à des occasions antérieures, et d'éléments carrément non-scientifiques.

On peut s'imaginer très peu de sous-domaines de la linguistique appliquée où la linguistique populaire comme point de relais ne jouerait aucun rôle. Nous avons déjà mentionné les problèmes de communication généraux (intra- et interculturels), l'apprentissage des langues étrangères, mais aussi par exemple la politique linguistique. Comme il y a un continuum entre «théories scientifiques » et «théories populaires », il y a aussi un continuum entre " experts » et «non-experts », avec nombre d'étapes intermédiaires, que nous avons déjà évoqué plus haut. Quand des politiciens ou des managers se prononcent pour ou contre l'anglais comme seule langue internationale - s'agit-il là de linguistique appliquée, de linguistique populaire ou juste d'une conviction idéologique qui n'est pas directement « dérivable » de la linguistique elle-même ?

\section{Conclusion}

Il y a encore beaucoup de questions ouvertes quant au rôle et à la valeur qu'on veut attribuer à la linguistique populaire et comment on voit les rapports de celleci avec d'autres phénomènes potentiellement apparentés (comme "language awareness »). Ce serait utopique de croire qu' on arrivera un jour à une estimation homogène et unitaire de la linguistique «non officielle ». Mais aujourd'hui il ne paraît plus si facile de voir la linguistique populaire uniquement comme linguistique défaillante et erronée, opposée à l'infaillible linguistique théorique, scientifique, officielle. Sans aucun doute, on peut et on doit critiquer certaines positions de la linguistique populaire (un certain purisme et « essentialisme» irréfléchis, etc.), mais surtout quand on a l'intention de contribuer à un «awareness raising » / une 
« conscientisation » général(e), à une amélioration de la formation linguistique et communicationnelle de nos citoyens / de nos citoyennes ${ }^{(1)}$, il faut prendre au sérieux leurs croyances populaires - il faut essayer de reconstruire les raisons pour lesquelles ils ont développé telle ou telle conviction. Avant de vouloir influencer des opinions problématiques, il faudra d'abord essayer de les comprendre, au moins d'une manière empathique.

\section{Bibliographie}

Achard-Bayle, G. \& PAveau, M.-A. (2008) : « La linguistique "hors du temple" », Pratiques 139-140, «Linguistique populaire? », ici même, pp. 3-16.

Antos, G. (1996) : Laien-Linguistik. Studien zu Sprach-und Kommunikationsproblemen im Alltag ; am Beispiel von Sprachratgebern und Kommunikationstrainings, Tübingen, Niemeyer.

BREKLE, H. E. (1985) : " "Volkslinguistik" : ein Gegenstand der Sprachwissenschaft bzw. ihrer Historiographie ? », Januschek, F., éd., Politische Sprachwissenschaft. Zur Analyse von Sprache als kulturelle Praxis, Opladen, Westdeutscher Verlag, pp. 145-156.

- (1986) : " Einige neuere Überlegungen zum Thema Volkslinguistik », Brekle, H. E. / Maas, U., Sprachwissenschaft und Volkskunde. Perspektiven einer kulturanalytischen Sprachbetrachtung, Opladen, Westdeutscher Verlag, pp. 70-76.

— (1989) : «La linguistique populaire», Auroux, S. (éd.), Histoire des idées linguistiques, Bruxelles, Mardaga, tome 1, pp. 39-44.

BRUMFIT, C. (1997) : "How Applied Linguistics is the same as any other science. », International Journal of Applied Linguistics 7/1, pp. 86-94.

CANDELIER, M. (éd.) (2003) : Janua Linguarum. La porte des langues. L'introduction de l'éveil aux langues dans le curriculum, Strasbourg, Conseil de 1'Europe.

Chryshochoos, N. (1992) : "Learners' awareness of their learning », James, Garrett, pp. 148-162.

CiCHON, P. (2005) : " "Falsches" und "fehlendes" Sprachbewusstsein - brauchbare oder irreführende Erklärungsbegriffe ?», Quo vadis Romania 25, pp. 13-19.

Donmall, B.G. (ed.) (1985) : Language Awareness, NCLE Reports and Papers, 6, London : CILT.

EDMONDSON, W. J. (1997) : « Sprachlernbewußtheit und Motivation beim Fremdsprachenlernen ", Fremdsprachen Lehren und Lernen 26, pp. 88-110.

FURnHAM, A. F. (1988) : Lay Theories. Everyday understanding of problems in the social sciences, Oxford, Pergamon Press.

Garrett, P., COUPland, N., Williams, A. (2003) : Investigating language attitudes : social meanings of dialect, ethnicity and performance, Cardiff, University of Wales Press.

(1) NdÉ : cf. F. Capucho (ici même, pp. 238-250). 
Gnutzmann, C. (2003) : "Language Awareness, Sprachbewusstheit, Sprachbewusstsein », Bausch, K.-R., Christ, H., Krumm, H.-J. (éds), Handbuch Fremdsprachenunterricht, Tübingen, Basel, Francke, pp. 335-339.

Groeben, N. et al. (1988) : Forschungsprogramm Subjektive Theorien. Eine Einführung in die Psychologie des reflexiven Subjekts, Tübingen, Francke.

HaWKINS, E. (1987) : Awareness of language: An introduction, New York, Cambridge University Press.

JAmes, C., GARrett, P. (eds.) (1992) : Language Awareness in the Classroom, London, Longman.

JESSNER, U. (2006) : Linguistic Awareness in Multilinguals, Edinburgh, Edinburgh University Press.

KALlENBACH, C. (1996): Subjektive Theorien. Was Schüler und Schülerinnen über Fremdsprachenlernen denken, Tübingen, Narr.

KnAPP-Potthoff, A. (1997) : "Sprach(lern)bewußtheit im Kontext », Fremdsprachen Lehren und Lernen 26, pp. 9-23.

MEIBNER, F.-J. (2003) : « Didactique du plurilinguisme et didactique pluriculturelle», en ligne sur http ://www.uni-giessen.de/meissner/meissner/msdf.htm.

MEIßNER, F.-J., REINFRIED, M. (éds) (1998) : Mehrsprachigkeitsdidaktik. Konzepte, Analysen, Lehrerfahrungen mit romanischen Fremdsprachen, Tübingen, Narr.

MORKÖTTER, S. (2005) : Language Awareness und Mehrsprachigkeit. Eine Studie zur Sprachbewusstheit und Mehrsprachigkeit aus der Sicht von Fremdsprachenlernern und Fremdsprachenlehrern, Frankfurt, Lang.

PAVEAU, M.-A. (2006) : «Linguistique populaire et enseignement de la langue : des catégories communes ? », Le Français aujourd'hui 151, pp. 95-107.

— (2007) : «Les normes perceptives de la linguistique populaire », Langage et Société, 121, pp. 93-109.

- (2008) : «Les non-linguistes font-ils de la linguistique ? Une approche antiéliminativiste des théories folk », Pratiques 139-140, "Linguistique populaire ? », ici même, pp. 93-109.

RoBinson, P. (1995) : "Attention, memory and the noticing hypothesis », Language Learning 45(2), pp. 283-331.

SCHMIDT, R. (éd.) (1995) : Attention and awareness in foreign language teaching and learning, Honolulu, University of Hawai'i at Manoa.

SCHÜTZ, A. (1932) : Der sinnhafte Aufbau der sozialen Welt. Eine Einleitung in die verstehende Soziologie, Wien, Springer.

Stegu, M. (1998) : Postmoderne Semiotik und Linguistik. Möglichkeiten, Anwendungen, Perspektiven, Frankfurt am Main, Lang.

— (2007) : «Der / die "ideale" Fachsprachenlehrende im Spannungsfeld "ExpertIn- Laie" », Engberg. J., Grove Ditlevsen, M., Kastberg, P., Stegu, M. (éds), New Directions in LSP Teaching, Bern, Lang, pp. 17-39.

- (2008a) : «Norm awareness », paper held at the $9^{\text {th }}$ Conference of the Association of Language Awareness, Hong Kong (unpublished).

— (2008b (à paraître) : « Code switching awareness : Zwischen welchen Codes kann, darf und soll geswitcht werden ? », Lavric, E., Kuhn, J., Fischer, F., Wochele, H. (éds), Language, products and professions. From code choice to onomastics, Frankfurt am Main, Lang.

TOMAlin, B., STEMPlEwski, S. (1993) : Cultural Awareness, Oxford, Oxford University Press. 